\title{
Generalized Characters Over Numerical Fields and a Counterpart of Chudakov Hypothesis
}

\author{
V. A. Matveev, O. A. Matveeva
}

Saratov State University, 83, Astrakhanskaya str., 410012, Saratov, Russia, vladimir.matweev@gmail.com, olga.matveeva.0@gmail.com

The well-known Chudakov hypothesis for numeric characters, conjectured by Chudakov in 1950, suggests that finite-valued numeric character $h(n)$, which satisfies the following conditions: 1) $h(p) \neq 0$ for almost all prime $p$; 2) $S(x)=\sum_{n \leq x} h(n)=\alpha x+O(1)$, is a Dirichlet character. A numeric character which satisfies these conditions is called a generalized character, principal if $\alpha \neq 0$ and non-principal otherwise. Chudakov hypothesis for principal characters was proven in 1964, but for non-principal ones thus far it remains unproved. In this paper we present a definition of generalized character over numerical fields, suggest an analog of Chudakov hypothesis for these characters and provide its proof for principal generalized characters.

Key words: Chudakov hypothesis, generalized numerical characters.

\section{References}

1. Kheil'bronn H. $\zeta$-funktsii i $L$-funktsii [ $\zeta$-functions and $L$-functions]. Algebraicheskaia teoriia chisel [Algebraic Number Theory]. Moscow, Mir, 1968, pp. 310-346 (in Russian).

2. Chudakov N. G., Linnik J. A. On certain class of completely multiplicative functions. USSR AS Reports, 1950, vol. 74, iss. 2, pp. 193-196 (in Russian).

3. Chudakov N. G., Rodosskii K. A. On generalized character. USSR AS Reports, 1950, vol. 74, iss. 3, pp. 1137-1138 (in Russian).

4. Glazkov V. V. Characters of multiplicative semigroup of natural numbers. Number theory research: Interacademic tractate collection, Saratov, Saratov Univ. Press, 1968, vol. 2, pp. 3-40 (in Russian).

5. Kuznetsov V. N., Setsinskaia E. V., Krivobok V. V. On a problem of decomposition into a product of Dirichlet

$L$-functions over numerical fields. Chebyshevskii sbornik [Chebyshev collection], 2004, vol. 5, iss. 3, pp. 51-64 (in Russian).

6. Vodolazov A. M., Kuznetsov V. N. On analytical continuation of Dirichlet series with multiplicative coefficients. Research on algebra, number theory and complementary areas: Interacademic tractate collection, Saratov, Saratov Univ. Press, 2003, iss. 1, pp. 43-59 (in Russian).

7. Dem'ianov V. F., Malozemov V. N. Vvedenie v minimaks [Introduction to minimax]. Moscow, Nauka, 1982, 368 p. (in Russian).

8. Postnikov A. G. Vvedenie v analiticheskuiu teoriiu chisel [Introduction to analytical number theory]. Moscow, Nauka, 1971, 416 p. (in Russian).

УДК 501.1

\section{О ПОЧТИ ПЕРИОДИЧЕСКИХ НА БЕСКОНЕЧНОСТИ РЕШЕНИЯХ РАЗНОСТНЫХ УРАВНЕНИЙ}

\author{
А. А. Рыжкова ${ }^{1}$, И. А. Тришина ${ }^{2}$ \\ ${ }^{1}$ Аспирантка касредры нелинейных колебаний, Воронежский государственный университет, anna-ryzhkova212@ rambler.ru \\ ${ }^{2}$ Аспирантка кафредры нелинейных колебаний, Воронежский государственный университет, I.A.Trishina@gmail.com \\ Введен в рассмотрение класс почти периодических на бесконечности последовательностей. Необходимость исследования \\ таких последовательностей связана с тем, что они возникают при рассмотрении разностных уравнений. Основные результаты \\ статьи связаны с доказательством почти периодичности на бесконечности решений разностных уравнений.
}

Ключевые слова: периодические на бесконечности последовательности, разностные уравнения, спектральная теория.

\section{ВВЕДЕНИЕ}

Пусть $\mathbb{Z}-$ множество целых чисел и - комплексное банахово пространство. Через $l^{\infty}=l^{\infty}(\mathbb{Z}, X)$ обозначим банахово пространство ограниченных последовательностей $x: \mathbb{Z} \rightarrow X$ с нормой $\|x\|_{\infty}=\sup _{n \in \mathbb{Z}}\|x(n)\|$. Через $c_{0}$ обозначим (замкнутое) подпространство последовательностей из $l^{\infty}$, убывающих на бесконечности, т. е. $\lim _{|n| \rightarrow \infty}\|x(n)\|=0, x \in c_{0}$. В пространстве $l^{\infty}$ рассмотрим операторы сдвига $S(n): l^{\infty} \rightarrow l^{\infty},(S(n) x)(k)=x(k+n), k \in \mathbb{Z}, n \in \mathbb{Z}, x \in l^{\infty}$. Используемые результаты из гармонического анализа, функции и векторов, содержатся в работах [1-7]. Следуя [1, 6, 7], дадим определение медленно меняющейся на бесконечности последовательности. 
Определение 1. Последовательность $x \in l^{\infty}$ называется медленно меняющаяся на бесконечности, если $S(1) x-x \in c_{0}$, т. е. $\lim _{n \rightarrow \infty}\|x(n+1)-x(n)\|=0$.

Примером медленно меняющейся на бесконечности последовательности является последовательность $x: \mathbb{Z} \rightarrow \mathbb{C}$ вида

$$
x(n)=\sin (\ln (\alpha+|n|)), \quad n \in \mathbb{Z}, \quad \alpha>0 .
$$

Множество медленно меняющихся на бесконечности последовательностей образует замкнутое подпространство из $l^{\infty}$, которое обозначим символом $l_{s l, \infty}^{\infty}=l_{s l, \infty}^{\infty}(\mathbb{Z}, X)$.

В статье [6] было введено понятие почти периодической на бесконечности функции. Такие функции использовались для описания ограниченных решений дифференциальных уравнений в банаховом пространстве. При рассмотрении разностных уравнений в данной статье будет использовано вводимое здесь понятие почти периодической последовательности. В отличие от [6] в основу определения положено определение Бора.

Определение 2. Пусть $\varepsilon>0$. Число $m \in \mathbb{Z}$ называется $\varepsilon$-периодом последовательности $x \in l^{\infty}(\mathbb{Z}, X)$ на бесконечности, если существует такое число $k \in \mathbb{N}$, что $\sup _{|n|>k}\|x(n+m)-x(n)\|<\varepsilon$. Множество $\varepsilon$-периодов последовательности $x$ на бесконечности обозначим через $\Omega_{\infty}(x, \varepsilon)$.

Определение 3. Множество $\Omega_{\infty}(x, \varepsilon)$ называется относительно плотным на $\mathbb{Z}$, если существует такое $l \in \mathbb{N}$, что $\{n, n+1, \ldots, n+l\} \cap \Omega_{\infty}(x, \varepsilon) \neq \varnothing$ для всех $n \in \mathbb{Z}$.

Определение 4 (определение Бора). Последовательность $x \in l^{\infty}(\mathbb{Z}, X)$ называется почти периодической на бесконечности, если для любого $\varepsilon>0$ множество $\Omega_{\infty}(x, \varepsilon)$ относительно плотно на $\mathbb{Z}$.

Множество почти периодических на бесконечности последовательностей обозначим символом $A P_{\infty}(\mathbb{Z}, X)$.

Теорема 1. Множество $A P_{\infty}(\mathbb{Z}, X)$ образует банахово пространство и банахову алгебру, если $X-$ банахова алгебра.

Доказательство. Если $x, y \in A P_{\infty}(\mathbb{Z}, X)$, то доказательство почти периодичности на бесконечности их суммы (произведения, если $X$ банахова алгебра) проводится точно по той же схеме, что и доказательство соответствующих свойств для почти периодических функуий (см. [8, § 1.2, свойство 6]). Докажем, что $A P_{\infty}(\mathbb{Z}, X)$ - банахова алгебра (с поточечным умножением последовательностей, если $X$ - банахова алгебра). Докажем полноту пространства $A P_{\infty}(\mathbb{Z}, X)$. Пусть $\left(x_{n}\right)$ - фундаментальная последовательность из $A P_{\infty}(\mathbb{Z}, X)$ и $x=\lim _{n \rightarrow \infty} x_{n}$ (используется полнота пространства $l^{\infty}$. Докажем, что $x \in A P_{\infty}(\mathbb{Z}, X)$. Пусть $\varepsilon>0$. Выберем натуральное число $N$ так, чтобы $\left\|x-x_{N}\right\|_{\infty}<\varepsilon / 4$. Рассмотрим множество $\Omega\left(\varepsilon / 4, x_{N}\right)$ ( $\left.\varepsilon / 4\right)$-периодов последовательности $x_{N}$. Следовательно, существует $k=k_{\varepsilon} \in \mathbb{N}$ такое, что $\sup _{|n|>k}\left\|x_{N}(n+m)-x_{N}(n)\right\|<\varepsilon / 4$ для любого $m \in \Omega\left(\varepsilon / 4, x_{N}\right)$. Тогда

$$
\begin{gathered}
\|x(n+m)-x(n)\| \leq\left\|x(n+m)-x_{N}(n+m)\right\|+\left\|x_{N}(n+m)-x_{N}(n)\right\|+\left\|x_{N}(n)-x(n)\right\| \leqslant \\
\leq \frac{\varepsilon}{4}+\frac{\varepsilon}{4}+\frac{\varepsilon}{4}=\frac{3 \varepsilon}{4}
\end{gathered}
$$

для всех $n \in \mathbb{Z}$, для которых $|n|>k$.

Поэтому

$$
\sup _{|n|>k}\left\|x_{N}(n+m)-x_{N}(n)\right\|<\varepsilon
$$

Таким образом, доказано включение $\Omega_{\infty}\left(\varepsilon / 4, x_{N}\right) \subset \Omega_{\infty}\left(\varepsilon, x_{N}\right)$, и, следовательно, множество $\varepsilon$-периодов последовательности $x$ относительно плотно на $\mathbb{Z}$.

Отметим, что банахово пространство $A P(\mathbb{Z}, X)$ почти периодических последовательностей содержится в $A P_{\infty}(\mathbb{Z}, X)$. 
Определение 5 (аппроксимационное). Последовательность $x \in l^{\infty}$ называется почтu neриодической, если для любого $\varepsilon>0$ существуют последовательности $x_{k} \in l_{s l, \infty}^{\infty}$ и числа $\gamma_{k} \in \mathbb{T}=\{\lambda \in \mathbb{C}:|\lambda|=1\}$ такие, что $\left\|x(n)-\sum_{k=0}^{N} x_{k}(n) \gamma_{k}^{n}\right\|<\varepsilon$.

Можно доказать, что эти определения (Бора и аппроксимационное) эквивалентны.

Ясно, что $l_{s l, \infty}^{\infty}(\mathbb{Z}, X) \subset A P_{\infty}(\mathbb{Z}, X)$. Это свойство отмечалось в статье [6].

Определение 6. Последовательность $x_{n}$ из $l^{\infty}(\mathbb{Z}, X)$ сходится $\kappa$ последовательности $x_{0} \in$ $\in l^{\infty}(\mathbb{Z}, X)$ на бесконечности, если для любого $\varepsilon>0$ можно указать число $n_{\varepsilon} \in N$ такое, что $\sup \left\|x(k)-x_{0}(k)\right\|<\varepsilon$. $|k| \geq n_{\varepsilon}$

Критерий (Бохнера). Функция $x \in l^{\infty}(\mathbb{Z}, X)$ является почти периодической на бесконечности тогда и только тогда, когда из любой последовательности сдвигов $\left(S\left(k_{n}\right) x\right), n>1$, можно вьделить подпоследовательность, сходящуюся на бесконечности к некоторой последовательности $x_{0} \in l^{\infty}$.

Лемма 1. Сумма и произведение двух почти периодических на бесконечности последовательностей есть почти периодическая последовательность.

Доказательство. Пусть $\left(\xi_{k}\right)$ - произвольная последовательность целых чисел. Выберем из нее такую подпоследовательность $\left(\xi_{k}^{\prime}\right)$, что последовательность последовательностей $S\left(\xi_{k}^{\prime}\right) x$ сходится, а затем из $\left(\xi_{k}^{\prime}\right)$ - такую подпоследовательность $\left(\xi_{k}^{\prime \prime}\right)$, что сходится подпоследовательность последовательностей $\left(S\left(\xi_{k}^{\prime \prime}\right) y\right.$. Тогда очевидно, что будет сходиться $S\left(\xi_{k}^{\prime \prime}\right)(x+y)$.

Теорема 2. Последовательность вида

$$
x(n)=\sum_{k=1}^{N} x_{k}(n) \gamma_{k}^{n}, \quad n \in \mathbb{Z}, \quad x: \mathbb{Z} \rightarrow X,
$$

где $\gamma_{k} \in \mathbb{T}, 1 \leq k \leq N, x_{k} \in l_{s l, \infty}^{\infty}(\mathbb{Z}, X)$, является почти периодической на бесконечности последовательностью $\left(x \in A P_{\infty}(\mathbf{Z}, X)\right)$.

Доказательство. По доказанному в теореме 1 каждая последовательность $x_{k}$ является почти периодической на бесконечности последовательностью. Любая периодическая на бесконечности последовательность является почти периодической на бесконечности последовательностью по определению. Последовательность $\widetilde{\gamma}: \mathbb{Z} \rightarrow \mathbb{C}, \widetilde{\gamma}(n)=\gamma^{n}$, где $n \in \mathbb{Z}, \gamma \in \mathbb{T}$, является также почти периодической последовательностью согласно критерию Бохнера. По доказанному в лемме 1 произведение почти периодической последовательности есть последовательность почти периодическая, следовательно, функция вида (2) является почти периодической на бесконечности последовательностью. Как отмечалось, $l_{s l, \infty}^{\infty}(\mathbb{Z}, X) \subset A P_{\infty}(\mathbb{Z}, X)$, а также $n \mapsto \gamma_{k}^{n}, 1 \leq k \leq n,-$ почти периодические последовательности.

\section{О ПОЧТИ ПЕРИОДИЧЕСКИХ НА БЕСКОНЕЧНОСТИ РЕШЕНИЯХ РАЗНОСТНОГО УРАВНЕНИЯ}

В банаховом пространстве $l^{\infty}(\mathbb{Z}, X)$, где $X$ конечномерное банахово пространство, рассмотрим разностное уравнение:

$$
x(n+N)=B x(n)+y(n), n \in \mathbb{Z},
$$

где $y \in c_{0}(\mathbb{Z}, X), B \in E n d X$ со свойством $\sigma_{0}=\sigma(B) \cap \mathbb{T}=\left\{\gamma_{1}, \gamma_{2}, \ldots, \gamma_{m}\right\}-$ совокупность простых собственных значений, где $\mathbb{T}=\{\lambda \in \mathbb{C}:|\lambda|=1\}$ и $\sigma(B)$ обозначает спектр оператора $B$.

Теорема 3. Каждое ограниченное решение $x: \mathbb{Z} \rightarrow X$ уравнения (1) является почти периодической на бесконечности последовательностью, которая допускает представление вида

$$
x(n)=\sum_{k=1}^{N} x_{k}(n) \gamma_{k}^{n},
$$

где $x_{k} \in l_{s l, \infty}^{\infty}, \gamma_{k} \in \mathbb{T}, 0 \leq k \leq N-1$ 
Доказательство. Спектр оператора В представим в виде

$$
\sigma(B)=\sigma_{0} \cup \sigma_{\text {in }} \cup \sigma_{\text {out }},
$$

где $\sigma_{0}=\sigma(B) \cap \mathbb{T}=\left\{\gamma_{1}, \gamma_{2}, \ldots, \gamma_{m}\right\}-$ совокупность собственных значений, лежащих на окружности; $\sigma_{i n}=\left\{\lambda \in \sigma(B):\left|\lambda_{j}\right|<1\right\}-$ совокупность собственных значений, лежащих внутри окружности $\mathbb{T}$; $\sigma_{\text {out }}=\left\{\lambda \in \sigma(B):\left|\mu_{j}\right|>1\right\}-$ совокупность собственных значений, лежащих вне окружности.

В соответствии с этим разбиением спектра рассмотрим проекторы $\mathscr{P}_{0}, \mathscr{P}_{i n}, \mathscr{P}_{\text {out }}$, которые соответственно построены по спектральным множествам $\sigma_{0}, \sigma_{\text {in }}, \sigma_{\text {out }}$. Таким образом, $I=\mathscr{P}_{0}+\mathscr{P}_{\text {in }}+\mathscr{P}_{\text {out }}$. Эти проекторы индуцируют разложение $X=X_{0} \oplus X_{i n} \oplus X_{\text {out }}$ пространства $X$, где $X_{0}=\operatorname{Im} \mathscr{P}_{0}$, $X_{\text {in }}=\operatorname{Im} \mathscr{P}_{\text {in }}, X_{\text {out }}=\operatorname{Im} \mathscr{P}_{\text {out }}$. Эти подпространства являются инвариантными для оператора В и пусть $B_{0}=B\left|X_{0}, B_{\text {in }}=B\right| X_{\text {in }}, B_{\text {out }}=B \mid X_{\text {out }}$. Таким образом, $B=B_{0} \oplus B_{\text {in }} \oplus B_{\text {out }}$ относительно построенного разложения пространства $X$. К обеим частям уравнения (1) применим проектор $\mathscr{P}_{i n}$, и тогда получим последовательность $x_{i n}=\mathscr{P}_{i n} x$, удовлетворяющую равенству

$$
S(N) x_{i n}(n)=B_{i n} x_{i n}(n)+y_{i n}(n),
$$

где $y_{\text {in }}=\mathscr{P}_{\text {in }} y \in c_{0}$.

Из (2) следует, что

$$
\left(I-B_{i n} S(-N)\right) x_{i n}=S(-N) y_{i n} .
$$

Поскольку $\|S(-N)\|=1, B_{i n} S(-N) x_{i n}(n)=S(-N) B_{i n} x_{i n}, n \in \mathbb{Z}$, и спектральный радиус $r\left(B_{i n}\right)$ оператора $B_{i n}$ меньше единицы, то оператор $I-B_{i n} S(-N)$ обратим и из (3) получаем, что $x_{i n}=\left(I-B_{i n} S(-N)\right)^{-1} S(-N) y_{i n}=\sum_{n=0}^{\infty} B_{i n}^{n} S(-N(n+1)) y_{i n}$. Ясно, что $x_{i n} \in c_{0}(\mathbb{Z}, X)$. Аналогичный результат получим при применении проектора $\mathscr{P}_{\text {out }}$ к уравнению (1):

$$
\left(S(N) x_{\text {out }}\right)(n)=B_{\text {out }} x_{\text {out }}(n)+y_{\text {out }}(n), \quad y_{\text {out }}=\mathscr{P}_{\text {out }} y \in c_{0} .
$$

Оператор $B_{\text {out }}$ обратим, и $\sigma\left(B_{\text {out }}^{-1}\right)=\left\{1 / \lambda_{j}, \lambda_{j} \in \sigma_{\text {out }}\right\}$, т. е. его спектральный радиус меньше единицы. Используя перестановочность оператора $S_{N}$ с $B_{\text {out }}$ из (4), получим равенства

$$
S(N) B_{\text {out }}^{-1} x_{\text {out }}(n)=x_{\text {out }}(n)+B_{\text {out }}^{-1} y_{\text {out }}(n), \quad n \in \mathbb{Z},
$$

или

$$
\left(I-S(N) B_{\text {out }}^{-1}\right) x_{\text {out }}(n)=-B_{\text {out }}^{-1} y_{\text {out }}(n), \quad n \in \mathbb{Z} .
$$

Таким образом,

$$
x_{\text {out }}(n)=-\left(I-S(N) B_{\text {out }}^{-1}\right)^{-1} B_{\text {out }}^{-1} y_{\text {out }}(n)=-\sum_{n=0}^{\infty}\left(B_{\text {out }}^{-1} S(N)\right)^{k} B_{\text {out }}^{-1} y_{\text {out }}, \quad y_{\text {out }} \in c_{0} .
$$

Из этой формулы следует, что $x_{\text {out }} \in c_{0}(\mathbb{Z}, X)$. Проектор $P_{0}$ можно представить в виде

$$
P_{0}=P_{1}+\ldots+P_{N}
$$

где $P_{k}-$ проектор, и

$$
A P_{k}=\gamma_{k} P_{k},
$$

где $\left|\gamma_{k}\right|=1,1 \leq k \leq N$.

Ввиду предполагаемой простоты собственных значений число $\gamma_{k}$ представимо в виде $\gamma_{k}=e^{i \lambda_{k}}$, $1 \leq k \leq N$. Применим проектор $P_{0}$ к разностному уравнению (1) и далее применим проектор $P_{k}$ :

$$
P_{k} x_{0}(n+1)=P_{k} B_{0} x_{0}(n)+P_{k} y_{0}(n),
$$

где $x_{0}=P_{0} x_{n}, x_{k}(n)=P_{k} x_{0}(n), k=\overline{1, N}$.

Следовательно, $x_{k}(n+1)=\gamma_{k} x_{k}(n)+y_{k}(n)$, где $x_{k}(n)=P_{k} x_{0}(n), k=\overline{1, N}, n \in \mathbb{Z}$. Сделав замену $x_{k}(n)=\gamma_{k}^{-n} x_{k}(n), n \in \mathbb{Z}$, получим:

$$
\widetilde{x}_{k}(n+1)=\widetilde{x_{k}}(n)+\widetilde{y_{k}}(n), \quad n \in \mathbb{Z},
$$


$\widetilde{x_{k}}$ - медленно меняющаяся последовательность, а $x_{k}(n)$ отличается от $\widetilde{x_{k}}(n)$ по формуле $(1)$ на множитель $\gamma_{k}^{n}$, где $\gamma_{k}-$ корень из единицы. Поскольку $\widetilde{y_{k}} \in c_{0}$ и $S(1) \widetilde{x_{k}}-\widetilde{x_{k}} \in c_{0}$, следовательно, $\widetilde{x_{k}}$, где $1 \leq k \leq m,-$ медленно меняющаяся на бесконечности последовательность.

Работа выполнена при финансовой поддержке РФФИ (проекты № 13-01-00378, № 12-01-31196).

\section{Библиографический список}

1. Баскаков А. Г., Калужина Н. С. Теорема Берлинга для функций с существенным спектром из однородных пространств и стабилизация решений параболических уравнений // Матем. заметки. 2012. Т. 92, № 5. С. 643661. DOI: $10.4213 / \mathrm{mzm} 8963$.

2. Баскаков А. Г. Теория представлений банаховых алгебр, абелевых групп и полугрупп в спектральном анализе линейных операторов // СМФН. 2004. Т. 9. С. 3151.

3. Баскаков А. Г., Криштал И. А. Гармонический анализ каузальных операторов и их спектральные свойства // Изв. РАН. Сер. матем. 2005. Т. 69, № 3. С. 354. DOI: $10.4213 / \mathrm{im} 639$.

4. Баскаков А. Г. Гармонический анализ линейных операторов. Воронеж : Изд-во Воронеж. гос. ун-та, 1987.
5. Дуплищева А. Ю. О периодических на бесконечности решениях разностных уравнений // Вестн. ВГУ. Сер. Физика. Математика. 2012. № 1. С. 110-117.

6. Баскаков А. Г. Исследование линейных дифференциальных уравнений методами спектральной теории разностных операторов и линейных отношений // УМН. 2013. Т. 68, № 1(409). С. 77-128. DOI: 10.4213/rm9505.

7. Баскаков А. Г., Калужина Н. С., Поляков Д. М. Медленно меняющиеся на бесконечности полугруппы операторов // Изв. вузов. Математика. 2014. № 7. С. 314.

8. Левитан Б. М., Жиков В. В. Почти-периодические функции и дифференциальные уравнения. М. : Изд-во МГУ, 1978.

\title{
Almost Periodic at Infinity Solutions of Difference Equations
}

\begin{abstract}
A. A. Ryzhkova, I. A. Trishina
Voronezh State University, 1, Universitetskaya pl., 304006, Voronezh, Russia, anna-ryzhkova212@rambler.ru, I.A.Trishina@gmail.com

A class of sequences almost periodic at infinity is introduced and studied. The necessity to consider such sequences is based on the fact that they appear in difference equations under consideration. The main results relate to the proof of almost periodicity at infinity of solutions of difference equations.
\end{abstract}

Key words: periodic sequence at infinity, difference equations, spectral theory.

This work was supported by the Russian Foundation for Basic Research (projects no. 12-01-31196, no. 13-0100378).

\section{References}

1. Baskakov A. G.,Kaluzhina N. S. Beurling's theorem for functions with essential spectrum from homogeneous spaces and stabilization of solutions of parabolic equations. Math. Notes, 2012, vol. 92, iss. 5, pp. 587605. DOI: 10.1134/S0001434612110016.

2. Baskakov A. G. Theory of representations of Banach algebras, and abelian groups and semigroups in the spectral analysis of linear operators. J. Math. Sci. (N. Y.), 2006, vol. 137, no. 4, pp. 4885-5036. DOI: 10.1007/s10958-006-0286-4.

3. Baskakov A. G., Krishtal I. A. Harmonic analysis of causal operators and their spectral properties. Izv. Math., 2005, vol. 69, no. 3, pp. 439-486. DOI: 10.4213/im639. 4. Baskakov A. G. Garmonicheskii analiz lineinykh operatorov [Harmonic analysis of linear operators]. Voronezh, Voronezh Univ. Press, 1987 (in Russian).
5. Duplishheva A. Ju. Periodic on infinity solutions of difference equations. The Proceeding VSU. Ser. Physics. Mathematics, 2012, vol. 1, pp. 110-117 (in Russian).

6. Baskakov A. G. Investigation of linear differential equations by the methods of the spectral theory of difference operators and linear relations. Russ. Math. Surv., 2013, vol. 68, no. 1, pp. 69-116. DOI: 10.1070/RM2013v068n01ABEH004822.

7. Baskakov A. G., Kaluzhina N. S., Polyakov D. M. Slowly varying at infinity semigroup of operators. Russian Math. [Izvestiya VUZ. Matematika], 2014, no. 7, pp. 3-14. DOI: 10.3103/S1066369X14070019.

8. Levitan B. M., Gikov V. V. Pochti-periodicheskie funktsii $i$ differentsial'nye uravneniya [Almost-periodic functions and differential equations]. Moscow, Moscow Univ. Press, 1978, 204 p. (in Russian). 